\title{
ATmospheric LIDar (ATLID): European Space Agency Instrument Ready to Measure Aerosols and Thin Clouds in Atmosphere
}

\author{
João Pereira do Carmo ${ }^{1}$, Geraud de Villele ${ }^{2}$, Kotska Wallace ${ }^{1}$, Alain Lefebvre ${ }^{1}$, Kaustav Ghose ${ }^{1}$, \\ Thomas Kanitz ${ }^{1}$, François Chassat ${ }^{2}$, Bertrand Corselle ${ }^{2}$, Thomas Belhadj ${ }^{2}$ and Paolo Bravetti ${ }^{2}$ \\ 1 European Space Agency - ESTEC, Noordwijk, The Netherlands; joao.pereira.do.carmo@esa.int \\ 2 Airbus Defense and Space, Toulouse, France; geraud.devillele@airbus.com
}

\begin{abstract}
ATLID (ATmospheric LIDar) is the atmospheric backscatter LIDAR (Light Detection and Ranging) on board of the EarthCARE (Earth Cloud, Aerosol and Radiation Explorer) mission, the sixth Earth Explorer Mission of the ESA (European Space Agency) Living Planet Programme [1-5]. ATLID's purpose is to provide vertical profiles of optically thin cloud and aerosol layers, as well as the altitude of cloud boundaries [6-10]. In order to achieve this objective ATLID emits short duration laser pulses in the UV, at a repetition rate of $51 \mathrm{~Hz}$, while pointing in a near nadir direction along track of the satellite trajectory. The atmospheric backscatter signal is then collected by its $620 \mathrm{~mm}$ aperture telescope, filtered through the optics of the instrument focal plane assembly, in order to separate and measure the atmospheric Mie and Rayleigh scattering signals. With the completion of the full instrument assembly in 2019, ATLID has been subjected to an ambient performance test campaign, followed by a successful environmental qualification test campaign, including performance calibration and characterization in thermal vacuum conditions. In this paper the design and operational principle of ATLID is recalled and the major performance test results are presented, addressing the main key receiver and emitter characteristics. Finally, the estimated instrument, in-orbit, flight predictions are presented; these indicate compliance of the ALTID instrument performance against its specification and that it will meet its mission science objectives for the EarthCARE mission, to be launched in 2023.
\end{abstract}

Keywords: LIDAR; UV laser; high spectral resolution; aerosols

\section{Introduction: The EarthCARE mission}

EarthCARE [1] is a joint collaborative mission of ESA and JAXA (Japan Aerospace Exploration Agency) with the objective to improve our understanding of the cloud-aerosol-radiation interactions and Earth radiative balance, so that they can be modelled with better reliability in climate and numerical weather prediction models. To provide atmospheric observations globally, EarthCARE satellite, Figure 1, will be placed in a Sun-Synchronous Orbit with a repeat cycle of 25 days. A low average altitude to ground of $408 \mathrm{~km}$ has been selected in order to enhance the performance of the two active instruments. The quasi-polar orbit will allow to cover all latitudes from equator to $\pm 83^{\circ}$. 


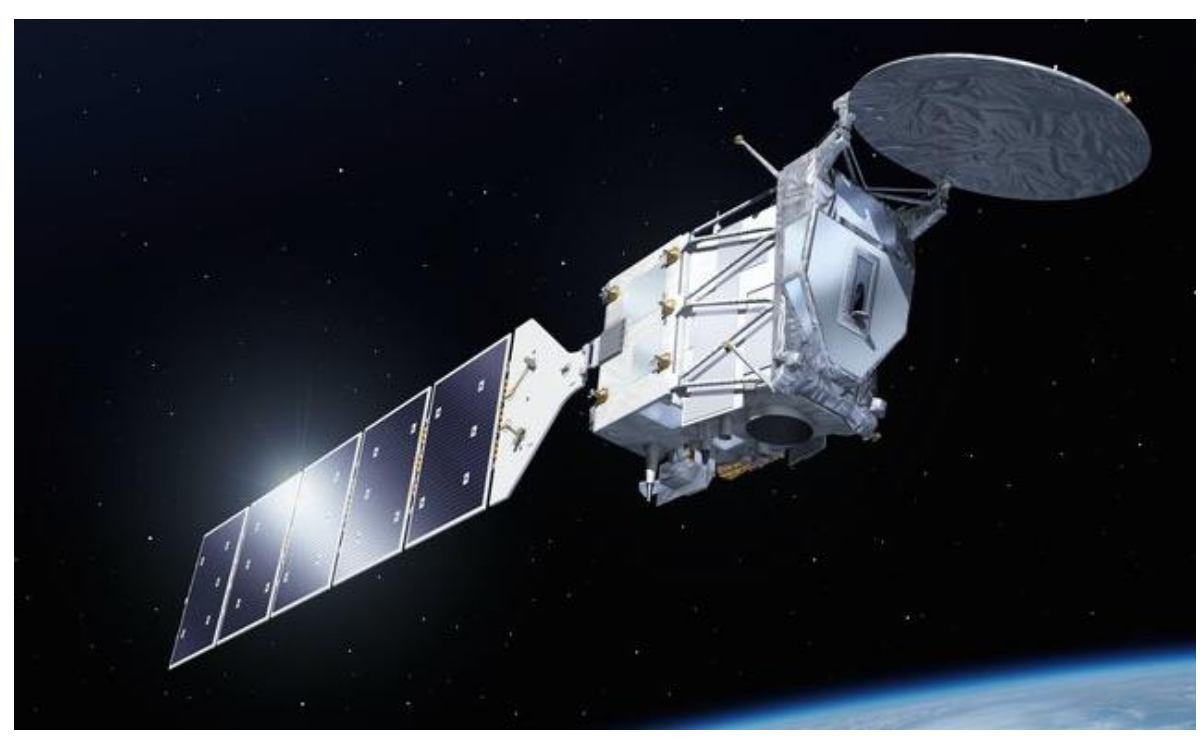

Figure 1. Artist's view of the EarthCARE satellite in-orbit.

The EarthCARE payload [2-5] consists of four instruments that will, in a synergetic manner, retrieve vertical profiles of clouds and aerosols, and the characteristics of the radiative and micro-physical properties, to determine flux gradients within the atmosphere as well as top of atmosphere radiance and flux. Specifically, EarthCARE scientific objectives are:

- Observation of the vertical profiles of natural and anthropogenic aerosols on a global scale, their radiative properties and interaction with clouds;

- Observation of the vertical distributions of atmospheric liquid water and ice on a global scale, their transport by clouds and their radiative impact;

- Observation of cloud distribution ('cloud overlap'), cloud precipitation interactions and the characteristics of vertical motions within clouds;

- Retrieval of profiles of atmospheric radiative heating and cooling through the combination of the retrieved aerosol and cloud properties.

EarthCARE payload comprises three instruments provided by ESA: a High Spectral Resolution UV ATmospheric LIDar (ATLID) [6], a Multi-Spectral Imager (MSI) and a Broad-Band Radiometer (BBR) [11]; and the Cloud Profiling Radar (CPR) [12] with Doppler capability, provided by JAXA. Co-registration of the multi-instrument payload data is a key aspect for the mission. The instrument viewing geometry can be seen in Figure 2, which illustrates the satellite ground track, the CPR beam at normal nadir, the ATLID beam de-pointed backward by $3^{\circ}$ to reduce specular reflection, the across track swath of the MSI with its offset in the anti-sun direction to mitigate sun-glint and finally the 3 BBR views in nadir, forward and backward directions required to retrieve the top of atmosphere emitted flux. 


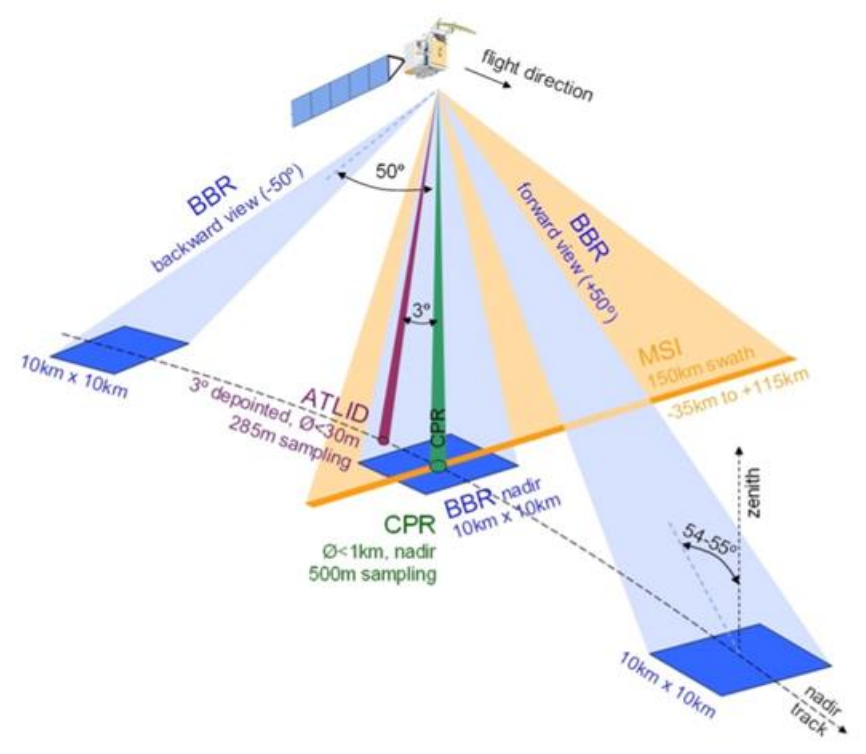

Figure 2. EarthCARE payload viewing geometry.

\section{ATLID measurement principle}

ATLID [9] is a high spectral Resolution LIDAR, operating in the ultra-violet spectral domain $(355 \mathrm{~nm})$, which takes benefit of the distinct interactions of light with molecules and aerosols that leads to different spectra scattering effects. Whereas the Brownian motion of molecules induces a wide broadening of the incident light spectrum, the scattering with an aerosol does not affect the spectral shape of the incident light. Consequently, a simple means of separating the backscattering contributions of aerosols and molecules consists of filtering the backscattered spectrum with a high spectral resolution filter centred on the laser emitted wavelength. In this way the instrument is able to separate the relative contribution of aerosol (Mie) and molecular (Rayleigh) scattering, which allows the retrieval of the aerosol optical depth. Co-polarised and cross-polarised components of the Mie scattering contribution are also separated and measured on dedicated channels. The operating wavelength in the UV spectral range was selected as the molecular scattering is high enough to measure more accurately extinction profiles and aerosols/thin clouds thickness and because laser technology (Nd:YAG laser with frequency tripling conversion) is available for operation in this spectral region. Additionally, eye safety versus field of view dimension were also taken into consideration for the selected operational wavelength.

As displayed in Figure 3, ATLID measures atmospheric profiles, in a direction close to the nadir, with a vertical resolution of about $100 \mathrm{~m}$ from ground to an altitude of $20 \mathrm{~km}$, and of $500 \mathrm{~m}$ from altitude $20 \mathrm{~km}$ to $40 \mathrm{~km}$. The instrument transmitter emits short laser pulses with a repetition rate of $51 \mathrm{~Hz}$, corresponding to about $285 \mathrm{~m}$ spatial sampling for a local accumulation of two shots, along the horizontal track of the satellite. Atmospheric, backscattered photons are collected by ATLID receiver using a $620 \mathrm{~mm}$ diameter telescope. 


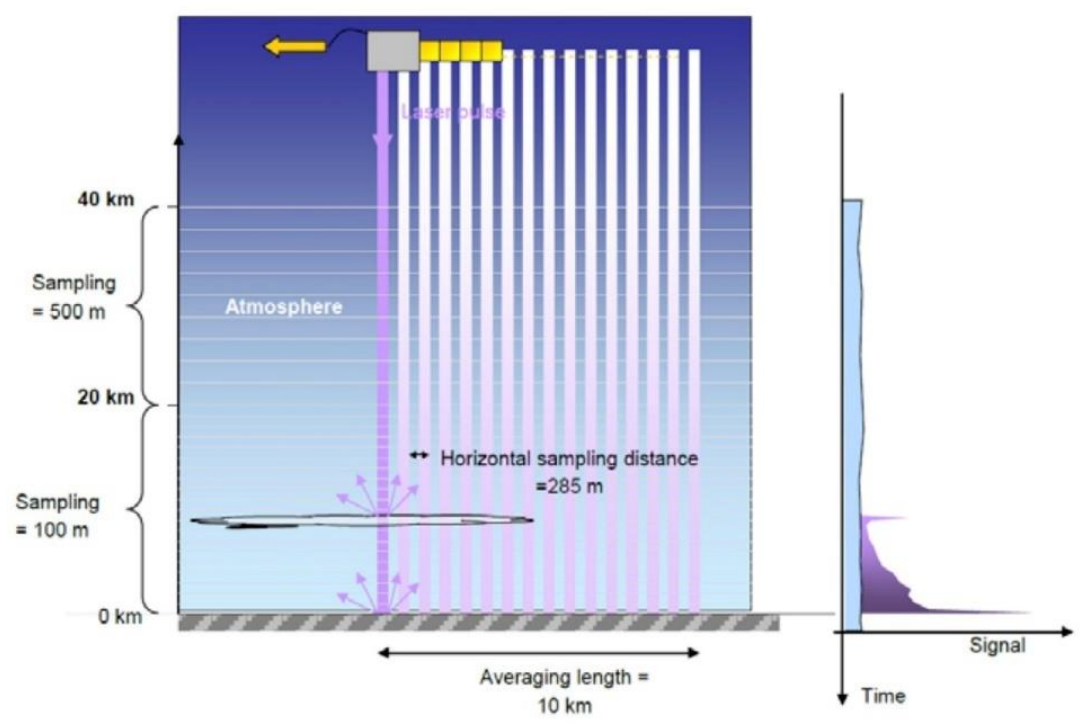

Figure 3. ATLID atmospheric measurement principle.

\section{ATLID instrument design}

Airbus Defence and Space in Toulouse was responsible for the design, assembly and test of ATLID. The instrument was designed as a self-standing instrument, as seen in Figure 4, thereby reducing the mechanical coupling between instrument / platform interfaces and allowing better flexibility in the satellite integration sequence.

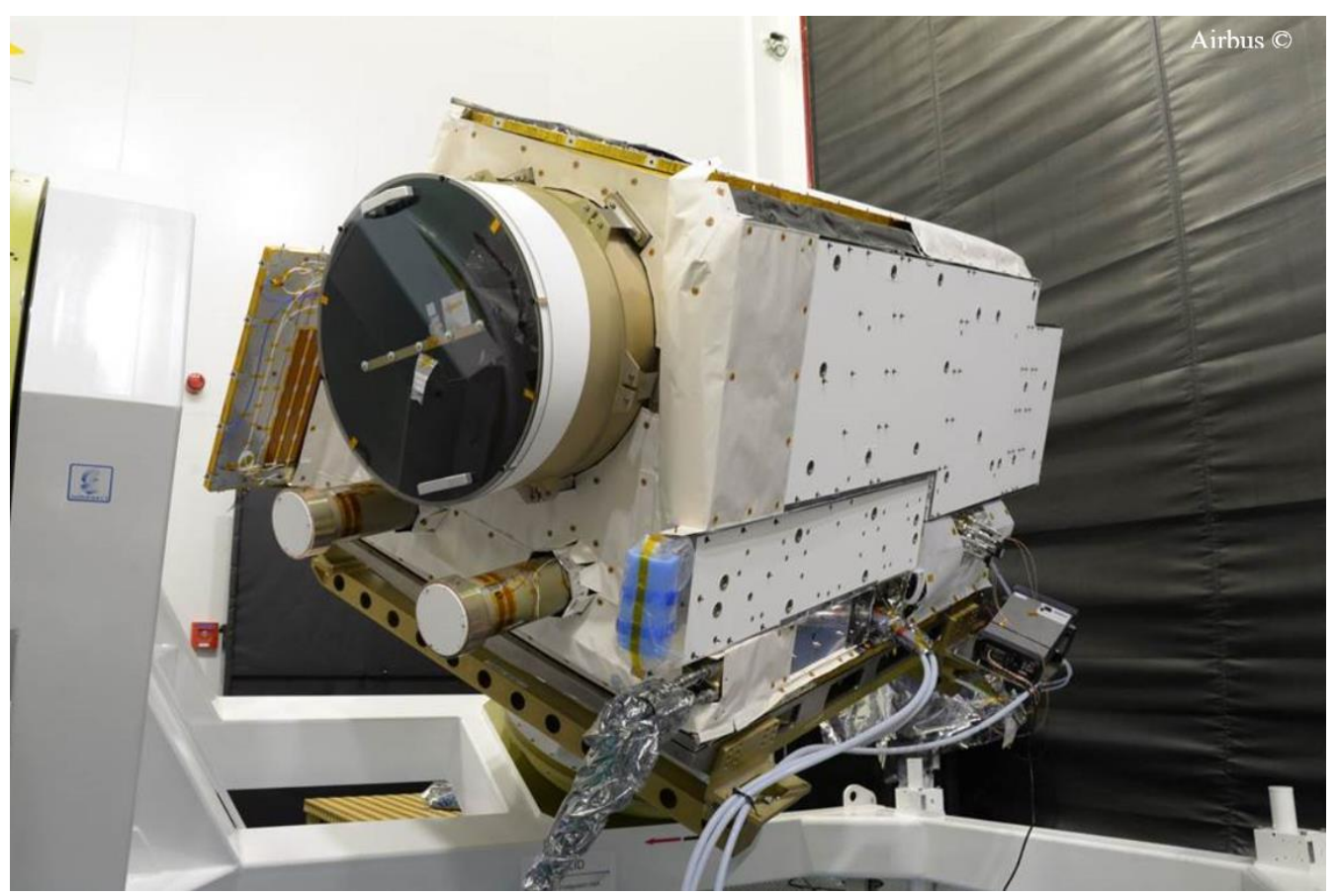

Figure 4. ATLID on trolley after completion of its integration.

ATLID instrument [7] development is based on a proto-flight model (PFM) approach: critical sub-systems such as laser transmitter, beam expander, beam steering mechanism and detector front-end have been subject to specific efforts in terms of early breadboards, electrical models or qualification models to minimise risks. The instrument is based on a bi-static architecture consisting of two independent main sections, the emitter chain and the receiver chain. ATLID functional architecture can be seen in Figure 5 . 


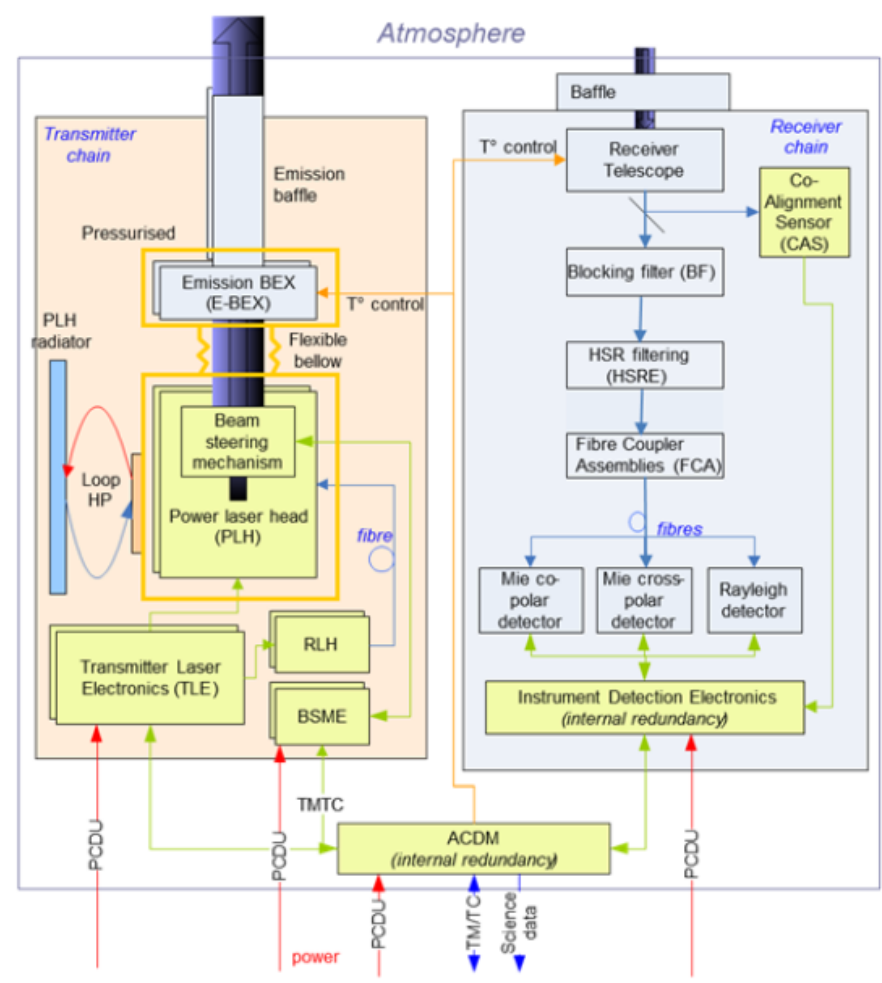

Figure 5. ATLID functional architecture.

On the emitter chain, the Transmitter Assembly (TxA), designed and manufactured by LEONARDO, is the laser source for the ATLID LIDAR. It comprises a Power Laser Head (PLH), seeded via a fibre optic by a Reference Laser Head (RLH), and its associated Transmitter Laser Electronics (TLE). There are two fully redundant transmitters (in cold redundancy), each including both laser heads (PLH and RLH) and electronics (TLE). The PLH design is based on a diode-pumped tripled Nd:Yag laser, providing a high energy pulse at $355 \mathrm{~nm}$. In the nominal measurement mode it is operated in steady-state mode with $51 \mathrm{~Hz}$ pulse repetition frequency (PRF). While the laser transmitter is largely inheriting from the Aladin instrument development for the AEOLUS mission [13], a significant evolution has been achieved by the fact that the ATLID PLH is sealed and pressurised in order to improve its tolerance to Laser Induced Contamination. Both TxA flight models, nominal and redundant units, demonstrated compliance with the main requirements: pulse energy $>35 \mathrm{~mJ}$, pulse duration $<35 \mathrm{~ns}$ and laser beam divergence $<300 \mu \mathrm{rad}(<45 \mu \mathrm{rad}$ after Emission Beam Expander).

At the heart of the receiver chain the High Spectral Resolution Etalon (HSRE), developed by RUAG Space, differentiates and filters the Mie and Rayleigh components of the backscatter signal, routing these (including splitting co-polarised from cross polarised light) towards the relevant detection channels. The key performance requirement of the HSRE is achieving high UV transmittance on the Mie co-polarised channel with a full-width-half-maximum (FHWM) of 0.3pm. The unit concept is based on a Fabry-Perot (FP) etalon, used in combination with polarisation beam-splitters (PBS) and quarter-wave plates. The Fabry-Perot etalon acts as a filter, transmitting only the narrow Mie signal and reflecting the wider Rayleigh signal.

The instrument implements a co-alignment control loop, making use of a Beam Steering Mirror (BSM) at transmitter chain level and a co-alignment sensor (CAS) at receiver chain level, in order to track and ensure that the emitted laser beam is aligned with the receiver field of view. This active alignment approach allows to correct for structural, thermo-elastic deformations that could be expected after launch or in orbit. 


\section{ATLID performance test overview}

\subsection{Performance verification approach}

The LIDAR performance verification consists of the analysis of recordings of the instrument receiver response to an atmospheric echo simulation and of the validation of instrument transmitter characteristics. The LIDAR instrument is tested in ambient for all performances that are not sensitive to air to vacuum effects: timing calibrations, detection alignment and functional operations, such as receiver to transmitter co alignment functions (Figure 6 right). Then final tests are performed in a thermal vacuum test chamber (Figure 6 left), where real flight conditions are reproduced; in this way performance is evaluated according to actual thermal control stability. Also, vacuum conditions can affect optical coating phase shifts, change their characteristics and impact instrument performance.

A limited set of key performance indicators are also measured at various stages of the satellite campaign on ground (in ambient conditions), in order to check the performance health of the ATLID, for instance upon delivery, after integration onto the satellite platform, before and after tests at satellite level. A dedicated in vacuum check of performance is also made during the satellite level thermal vacuum campaign.

\subsection{Receiver Measurement setup}

The instrument's high sensitivity to the simulated echo characteristics shall be discriminated from the possible bias or instabilities of the test setup. The simulated echo source is a tuneable laser coupled to an optical system that control its divergence, line of sight, wavelength, polarisation and pulse timings. Any uncontrolled variation of these parameters will induce a performance variation that will degrade the test accuracy.

The measurement setup used for the instrument characterisation is shown in Figure 6. It transmits a beam of the simplified atmospheric echo:

- Generated by a frequency-tripled, Nd:YAG, narrow-band OGSE (Optical Ground Support Equipment), UV laser (source-pack outside the vacuum chamber), which is fibre-coupled;

- Through part B of OGSE FPA (OGSE multi-purpose Focal Plane Assembly, outside the vacuum chamber, detailed in Figure 6), which allows for laser beam parameters adjustment, including polarisation, Line-Of-Sight and radiometric level, and then free path;

- Through OGSE collimator COL70 (70 $\mathrm{cm}$ diameter, inside the vacuum chamber); up to ATLID telescope input.
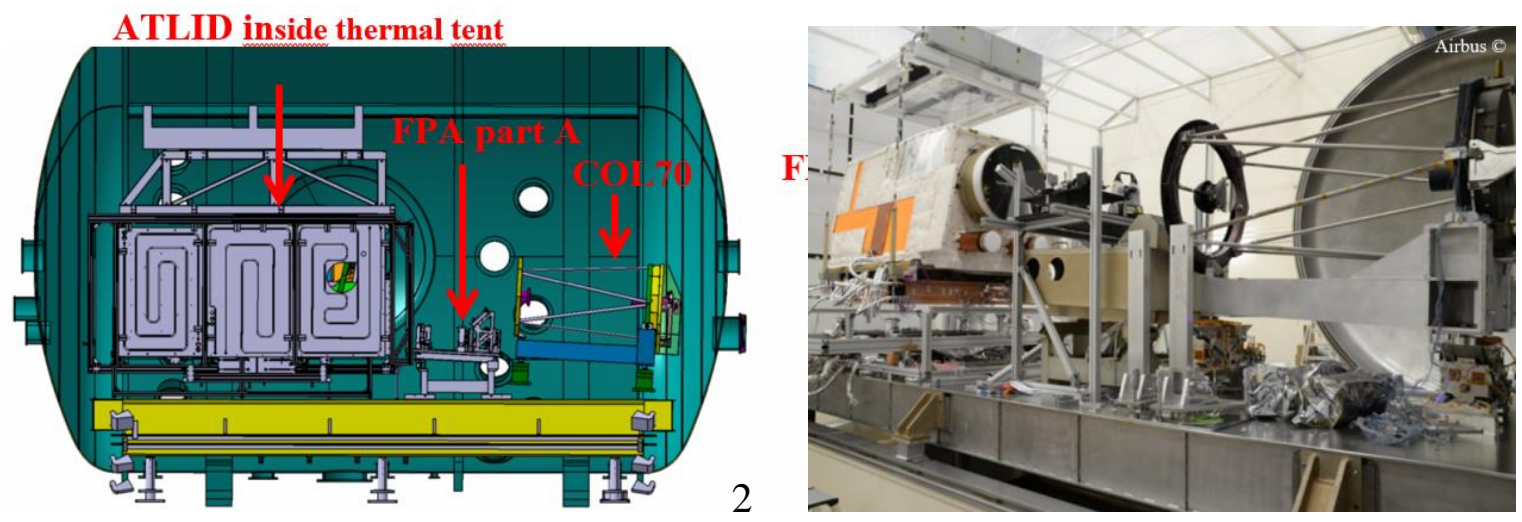

Figure 6. ATLID test configuration in vacuum chamber, with FPA (Focal Plane Assemblies) parts $\mathrm{A} \& \mathrm{~B}$ and collimator COL70.

\subsection{Transmitter Measurement setup}

The two ATLID emitters have been characterised in terms of:

1. Laser pulse energy 


\section{Laser beam divergence.}

The additional measurement setup used for the characterisation is shown in Figure 7 and indicated with FPA part A in Figure 6 (left). It can collect the radiation from both nominal and redundant lasers (yellow circles) into single path toward COL70 inside the vacuum chamber. Laser A beam (bottom circle) is reflected on front side of P0, while laser B beam (top circle) is going through prism $\mathrm{P0}$ and is reflected on back side. The wedge angle of this prism is sufficiently large to avoid any parasitic images within the FF (Far Field) image pattern $(<100 \mu \mathrm{rad})$. A camera system is placed at the output of COL70 system, outside the vacuum chamber on FPA part B, as per Figure 6 .
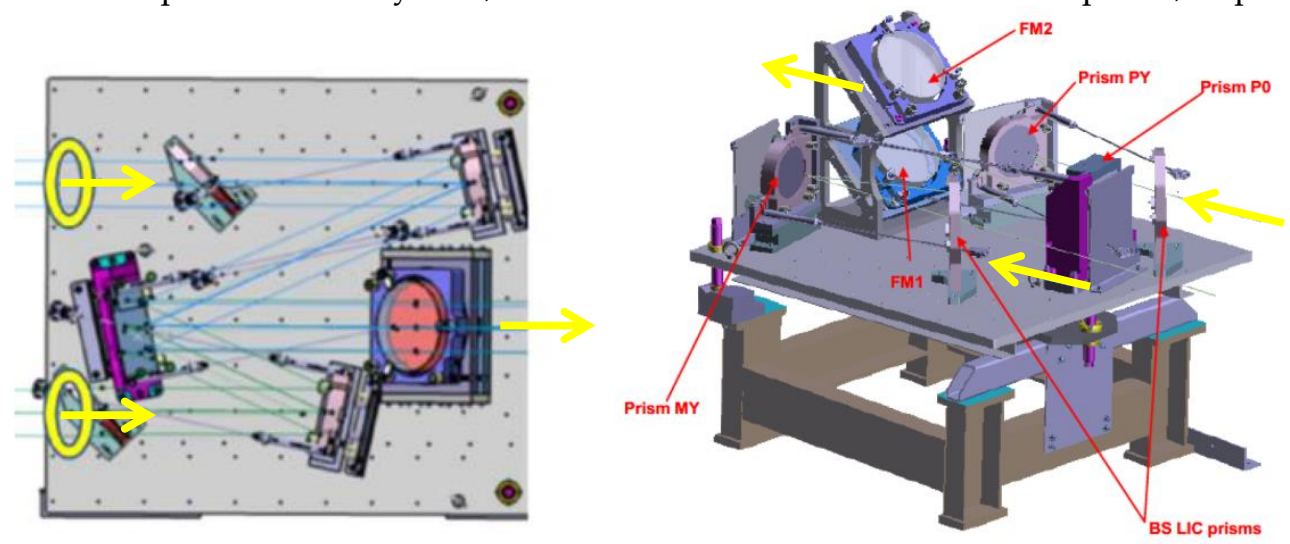

Figure 7. FPA part A, OGSE periscope setup for TxA beam collection into COL70.

\section{Test results}

\subsection{Transmitter test results}

\subsubsection{Laser pulse energy}

The objective was to estimate the energy knowledge via the internal laser photodiode monitoring. To this purpose, the absolute energy was measured before TVAC test, acquiring the entire laser beam spot. For TVAC test two additional photodiodes were installed and calibrated on the OGSE, one on its main bench (FPA part B) and one inside the vacuum chamber behind a partially reflective mirror (FPA part A). These two sensors aimed at providing a measurement reference external to the laser, all along the laser optical path, during the whole TVAC test.

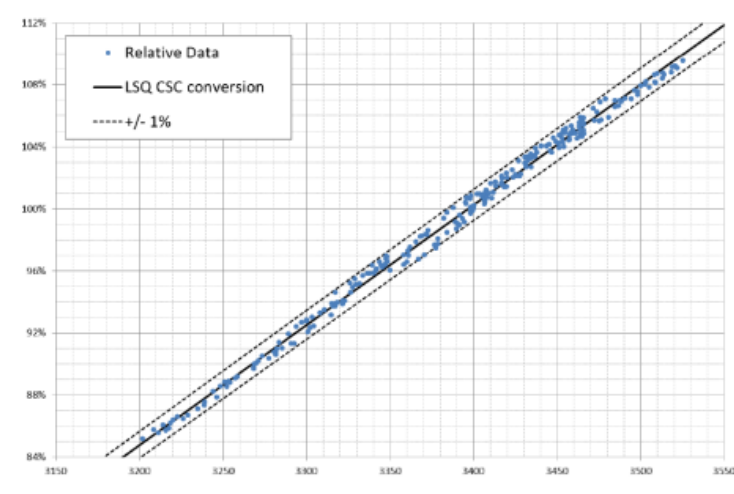

(a)

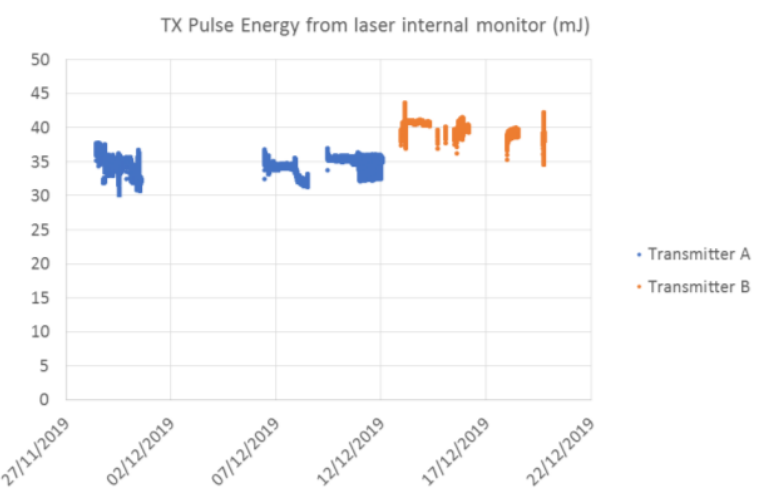

(b)

Figure 8. Example of Energy measurements performed during TVAC via the internal laser photodiode: (a) Energy telemetry calibration; (b) Overview of Energy measurements during the entire vacuum phase. 
An example result of the telemetry calibration is shown in Figure 8 (a). Figure 8 (b) shows the overall, internal photodiode reading during the entire test, for both emitters: energies between 35 and $40 \mathrm{~mJ}$ were reached.

\subsubsection{Laser beam divergence}

With this test a measurement of the Far Field Pattern (FFP) of the emitted laser beams was performed, thanks to the imaging capability of the COL70 collimator OGSE. The measurements were performed in vacuum, collecting OGSE images on a shot-to-shot basis (instantaneous FFP). Post processing averaging was done to compute integration over an equivalent of $10 \mathrm{~km}$ horizontal span. Additional measurements were also done to verify the capability to thermally adjust the External Beam Expander (EBEX) defocus and to quantify the thermal sensitivity; in this second phase, EDC (Emission Defocus Calibration) mode is commanded, inducing a slow scan of EBEX temperature. A special test procedure is used to optimise the number of steps (down to 4 or 5 steps) for this ground test, in order to reduce test duration compared to a finer stepping in flight.

Figure 9 shows the far field measurement results during EDC; as expected, the divergence is in the 15 - $45 \mu \mathrm{rad}$ range (with angular resolution lower than $2 \mu \mathrm{rad}$ and accuracy within $\pm 5 \mu \mathrm{rad}$ ). Far field pattern images are reported in the figure in correspondence to the different tests' EBEX temperatures.

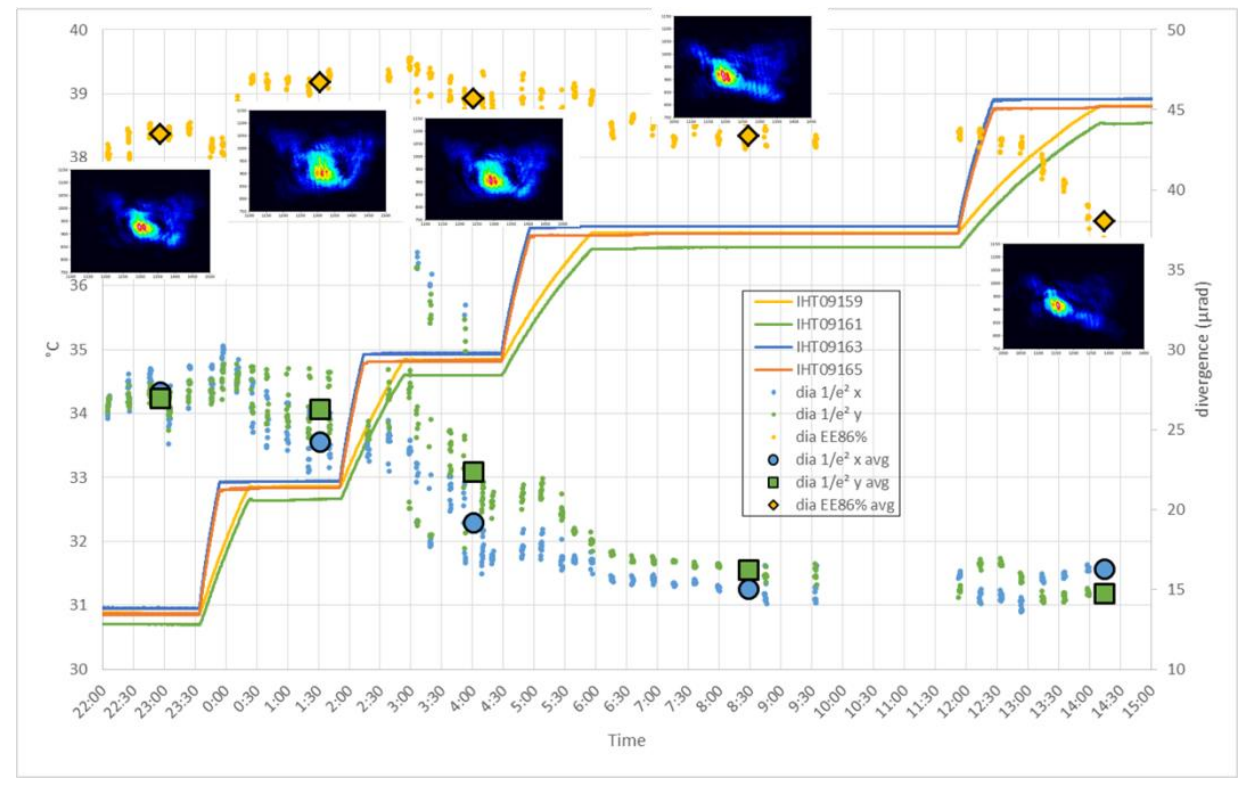

Figure 9. Far field measurement results during EDC; the variation of beam divergence (right scale) is measured and averaged for various EBEX temperatures (thermistor readings on the left scale).

The FFP was also acquired during the simulation of orbital cycle effects, i.e. for a $\pm 1{ }^{\circ} \mathrm{C}$ temperature oscillation on the transmitter thermal interface. Figure $\mathbf{1 0}$ shows the evolution of encircled energy divergence during about 6 cycles. The FF images corresponding to the minimum and maximum temperatures are reported inside the plot. 


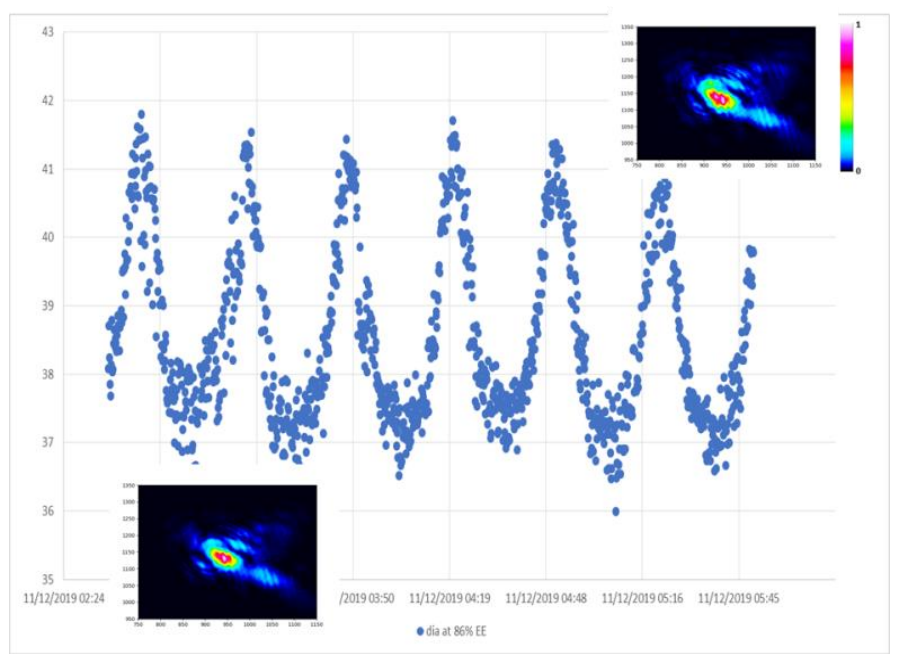

Figure 10. Far field measurement results during simulation of orbital cycles $\left( \pm 1^{\circ} \mathrm{C}\right.$ on the laser); the variation of beam divergence (right scale) is measured and averaged for various EBEX temperatures (thermistor readings on the left scale).

\subsection{Radiometric tests}

The ATLID receiver has been characterised in terms of:

- Polarisation

- Field-Of-View

- Radiometric stability

- Dark current

\subsubsection{ATLID receiver polarisation characterisation}

The objective here was to characterise the response of the ATLID receiver for each of the three channels, for both polarisation axes, and over at least one free spectral range of HSRE. To this purpose, the OGSE emission line-of-sight is centred in instrument Field-Of-View, radiometric level is adjusted to be in the instrument measurement dynamic, and:

- Scans of the OGSE laser wavelength are performed, for vertical and horizontal linear polarisation directions, and the channels' relative responses are extracted;

- Rotations of the OGSE beam linear polarisation orientation are performed, for OGSE source-pack laser wavelength set at HSRE response peak, and the channels' relative responses are extracted.

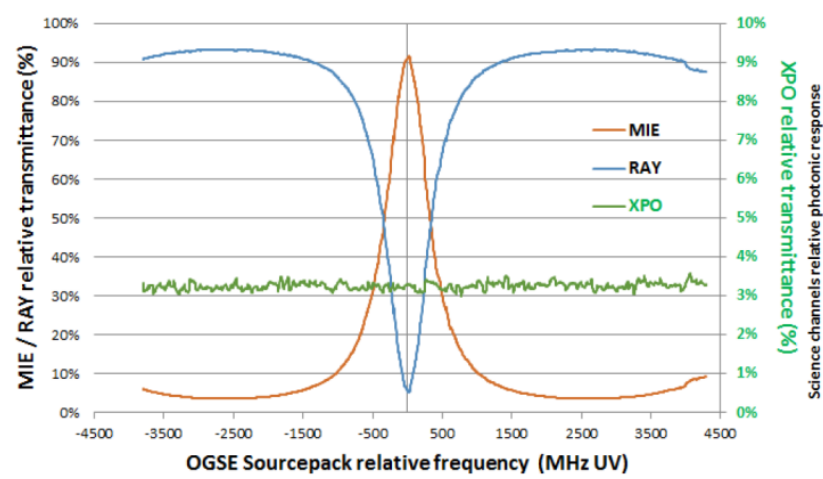

(a)

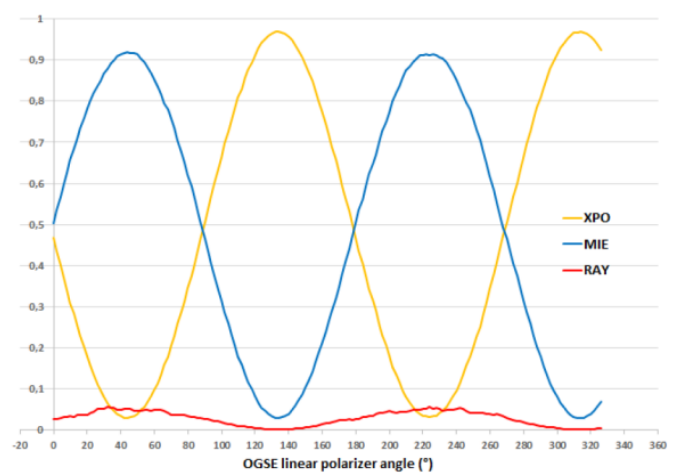

(b)

Figure 11. Example of polarisation measurements performed during TVAC; channels' relative responses versus: (a) OGSE frequency scan; (b) OGSE linear polarisation rotation 


\subsubsection{ATLID Field-Of-View characterisation}

The objective here was to evaluate the reception Field-of-View angular diameter, in order to assess the collecting solid angle of the instrument. The same test shall also permit to the reference receiver Line-of-Sight with respect to the co-alignment sensor. To this purpose, the OGSE emission line-of-sight is scanned around the centre of the instrument Field-of-View, radiometric level is adjusted to be in the instrument measurement dynamic, and the channels' responses are extracted and normalised. The angular width of the normalised response gives the angular diameter of the instrument reception Field-of-View, as can be seen in Figure 12.

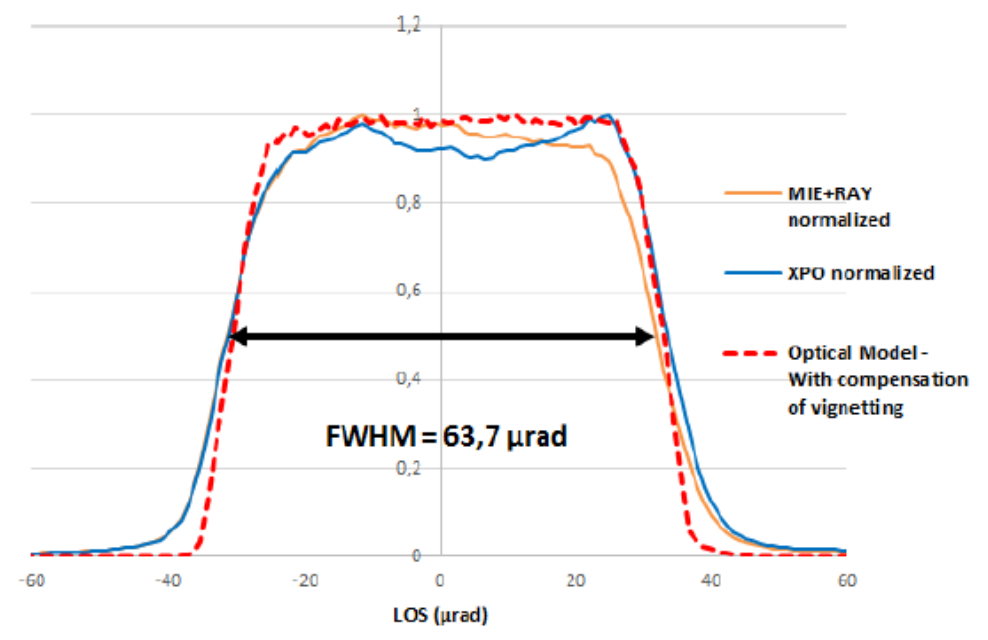

Figure 12. Example of instrument Field-of-View characterisation measurements performed during TVAC; channels' normalised responses vs OGSE Line-of-Sight scan

\subsubsection{ATLID radiometric stability characterisation}

The objective was to characterise the relative instrument response variation for each of the three science channels, with varying environment. To this purpose, the OGSE emission line-of-sight is centred in instrument Field-Of-View, OGSE laser wavelength is set at HSRE response peak and the thermal environment in the vacuum tank is managed in order to describe the equivalence of the foreseen orbital thermal cycle. The channels' signals are extracted and their stabilities during thermal cycle are calculated. As the OGSE frequency drift during the measurement contributes to signal variation during the test, its contribution is calculated and accounted for in the measured radiometric stability assessment, in order to assess the impact of thermal cycle only.

\subsubsection{ATLID dark current characterisation}

The objective was to record the dark signal map and the dark noise in operational conditions at a $-30^{\circ} \mathrm{C}$ setpoint for the detector CCD. To this purpose, the OGSE does not emit any signal and ATLID is in DCC mode (Dark Current Calibration). The output of this test allows filling the calibration database to be provided with the instrument and to be used by the EarthCARE ground processer. The dark noise all along the atmospheric profiles is below the defined 2.2 e- rms success criteria for all channels, validating the ATLID performance model budgeted value.

\section{ATLID performance update and flight prediction overview}

\subsection{Performance analysis}

The performance prediction is made using the test results obtained with an artificial, atmospheric echo simulation to reconstruct the instrument performance; as shown above during the instrument testing, no direct atmospheric test is performed. In particular, the test source spectrum is a monochromatic, monomode, longitudinal laser line at $355 \mathrm{~nm}$, while in flight the received 
backscattered signal spectrum will be broadened by Rayleigh backscattering superimposed onto a variable Mie spectrum line (see Figure 13).
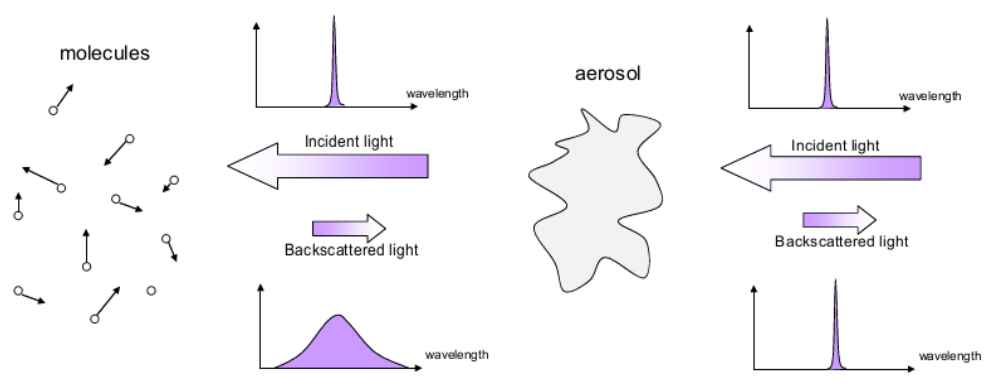

Figure 13. Illustration of the backscattered spectrum

Also, the echo will be partially depolarised by the atmospheric scene; for example, Sahara aerosols can depolarise up to $30 \%$ of the polarised light.

The instrument performance model is updated based on the instrument test results shown above. In particular, the receiver channel cross talks are verified and calibrated. This concerns at first the polarisation cross talk characteristic between co polar and cross polar channels that is checked via input beam of pure Co polarisation, or pure Cross polarisation. The spectral cross talk is also confirmed, by verifying the instrument spectrum response of the HSRE. The cross talk correction needs are shown in Figure 14.

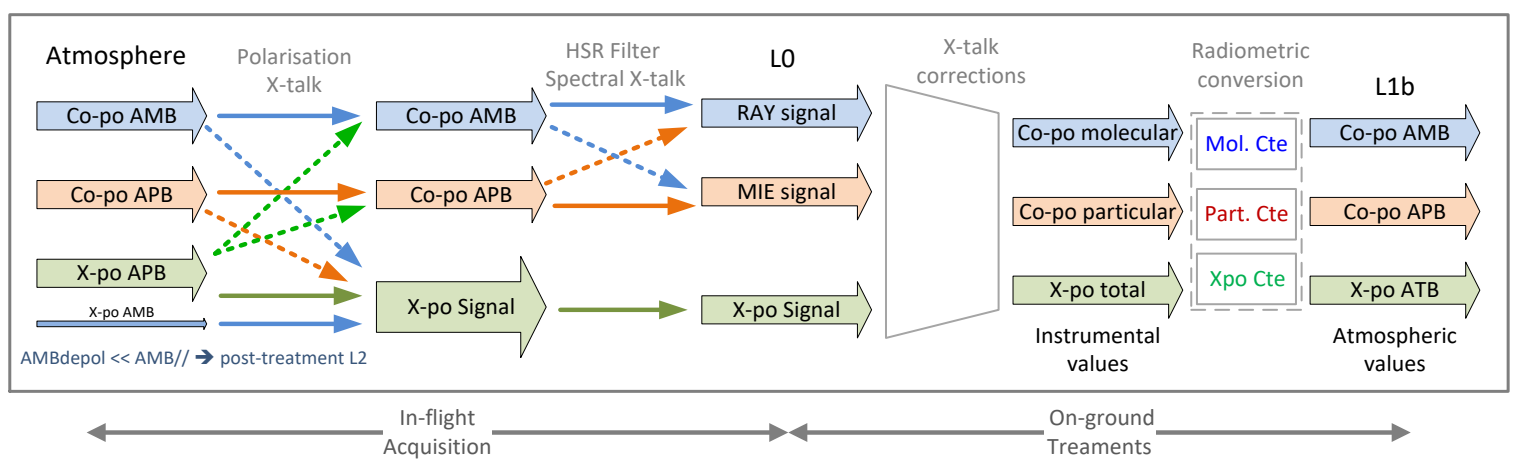

Figure 14. Illustration of signal cross talks and need for cross talk corrections.

\subsection{Performance summary}

Table 1 shows the ATLID instrument radiometric characteristics predicted for flight and Table 2 show the ATLID detection performance.

Table 3 shows the derived ATLID LIDAR performance of backscatter absolute retrieval accuracy, based on the relative retrieval accuracy on each channel and the absolute calibration of the LIDAR constant. The accuracy is given at $10 \mathrm{~km}$ altitude, on a $10 \mathrm{~km}$ horizontal integration length, for three types of scenes:

- a sub visible cirrus: faint cirrus at the limit of instrument detection, $\beta=8 \times 10^{-7} \mathrm{sr}^{-1} \mathrm{~m}^{-1}$

- a thin cirrus : $\beta=1.4 \times 10^{-5} \mathrm{sr}^{-1} \mathrm{~m}^{-1}$

- a depolarisation cirrus : cirrus with $10 \%$ depolarisation ratio, $\beta_{\mathrm{par}}=2.6 \times 10^{-5} \mathrm{sr}^{-1} \mathrm{~m}^{-1}$ and $\beta_{\mathrm{per}}=2.6 \times 10^{-6} \mathrm{sr}^{-1} \mathrm{~m}^{-1}$ 
Table 1. ATLID radiometric characteristics.

\begin{tabular}{|c|c|c|}
\hline Radiometric characteristics & ATLID & unit \\
\hline Receiver field of view & $66.5^{1}$ & $\mu \mathrm{rad}$ \\
\hline Telescope diameter & 620 & $\mathrm{~mm}$ \\
\hline Solar background filter equivalent bandwidth & 710 & pm \\
\hline Mie Co Polar transmission EOL & 45 & $\%$ \\
\hline Mie Cross Polar transmission EOL & 43 & $\%$ \\
\hline Rayleigh transmission EOL & 43 & $\%$ \\
\hline (molecular to molecular channel) C_mm ${ }^{3}$ & 75 & $\%$ \\
\hline (molecular to particle channel) C_mp ${ }^{3}$ & 25 & $\%$ \\
\hline (particular to molecular channel) C_pm ${ }^{3}$ & 16 & $\%$ \\
\hline (particular to particular channel) C_pp ${ }^{3}$ & 84 & $\%$ \\
\hline Laser pulse energy & 35 & $\mathrm{~mJ}$ \\
\hline Pulse repetition frequency & 51 & $\mathrm{~Hz}$ \\
\hline Transmitter field of view & 36 & $\mu \mathrm{rad}$ \\
\hline Transmitter EOL transmission & 89 & $\%$ \\
\hline Transmitter polarisation & Linear & - \\
\hline
\end{tabular}

${ }^{1}$ value predicted in flight is different from ground measured value due to gravity focusing effects;

2 End Of Life value without etalon transmission

${ }^{3}$ photo flux ratio characteristics of HSRE

Table 2. ATLID detection performance.

\begin{tabular}{ccc}
\hline Detection performance & ATLID & unit \\
\hline Vertical resolution from 0 to $20 \mathrm{~km}$ & 100 & $\mathrm{~m}$ \\
Vertical resolution from $20 \mathrm{~km}$ to $40 \mathrm{~km}$ & 500 & $\mathrm{~m}$ \\
Vertical cross talk & $4.5 \%$ on $500 \mathrm{~m}, 11 \%$ on $100 \mathrm{~m}$ & $\%$ \\
Computed quantum efficiency & $79 / 75 / 791$ & $\%$ \\
Linearity & $<+/-12$ & $\mathrm{e}-\mathrm{rms}$ \\
Noise worst case & $<+/-2^{3}$ & $\%$ \\
\hline
\end{tabular}

${ }^{1}$ (MieCoPo / MieCross / Rayleigh); ${ }^{2}$ from $10 \mathrm{e}-$ to the top; ${ }^{3}$ from $1 \mathrm{e}-$ to $10 \mathrm{e}-$

Table 3. ATLID LIDAR retrieval accuracy.

\begin{tabular}{cccc}
\hline $\begin{array}{c}\text { LIDAR absolute backscatter retrieval accuracy } \\
\text { 10km horizontal integration }\end{array}$ & Typical BOL & Worst Case EOL & unit \\
\hline Mie Co Polar on sub visible cirrus : $\beta=810^{-7} \mathrm{sr}^{-1} \mathrm{~m}^{-1}$ & 31 & 48 & $\%$ \\
Mie Co Polar on thin cirrus : $\beta=1.410^{-5} \mathrm{sr}^{-1} \mathrm{~m}^{-1}$ & 6 & 8 & $\%$ \\
Mie Cross polar on depol. cirrus : $\beta=2.610^{-6} \mathrm{sr}^{-1} \mathrm{~m}^{-1}$ & 19 & 23 & $\%$ \\
Rayleigh (above cirrus $10 \mathrm{~km})$ & 12 & 17 & $\%$ \\
\hline
\end{tabular}

\section{ATLID verification closure and lessons learnt}

Instrument performance verification steps have been completed as shown above. Following the environmental qualification and calibration at instrument level, ATLID has been integrated onto the EarthCARE satellite in March 2020 and will undergo satellite level thermal vacuum testing in 2022.

Lessons learnt from previous LIDAR experience on ALADIN have shown the importance of prediction of the long-term evolution of the laser properties. The lifetime elements of the laser are the $808 \mathrm{~nm}$ pump diode aging, the high fluence coatings and the laser housing pressure. The laser diodes have undergone life testing, to demonstrate their longevity in accordance with the requirements of ATLID flight unit ground testing and mission lifetime.

In addition, one other lesson learnt from ALADIN is the verification of the CCD dark current map stability. A "hot pixel" phenomena is observed on ALADIN, whereby random pixels exhibit an elevated signal level, with the number of hot pixels increasing over time [14]; this forces Aeolus satellite to have to compensate the effect with an additional, regular calibration that was not 
foreseen before flight. ATLID pixel level CCD behaviour has been characterised under flight-like operational parameters and the dark current values do not present the same level of defect, with no hot pixels seen; this is due to a different CCD control sequence, which reduces the time that the charge resides in the CCD by a factor of 10 . Nevertheless, the pixel responses will be monitored and checked again once in satellite thermal vacuum operation, where flight dark current value can be recorded with the nominal $-30^{\circ} \mathrm{C}$ operating temperature.

\section{Conclusions}

With the completion of the full instrument assembly in 2019 [8], ATLID has been subjected to an ambient performance test campaign $[9,10]$, followed by a successful environmental qualification test campaign, including performance calibration and characterisation in thermal vacuum conditions. ATLID has been successfully operated as a self-standing instrument and all performance aspects, including transmitter and receiver characteristics, have been found to be in line with targeted expectations and compliant with all major science, in-flight, end of life, performance requirements. This includes under worst case scenarios, where is assumed instrument performance degradation as well as operation in less favourable orbital points regarding sun illumination conditions. The instrument has been delivered to the EarthCARE industrial Prime for assembly and integration onto EarthCARE satellite platform, a task that was completed in May 2020. Instrument and platform interface verifications are now approaching an end, with the execution of the last performance checks and functional testing at system level. With the completion of the foreseen platform qualification test campaign and flight acceptance review in 2022, the launch of EarthCARE satellite in March 2023, and the first 6 months of in-orbit commissioning phase, ATLID will be ready to start its 3 year long mission, to measure aerosols and thin clouds from space. Data from ATLID is expected to provide a major contribution to the earth observation science community's understanding of the cloud-aerosol-radiation interaction and Earth radiative balance, and in development of more reliable climate and numerical weather prediction models

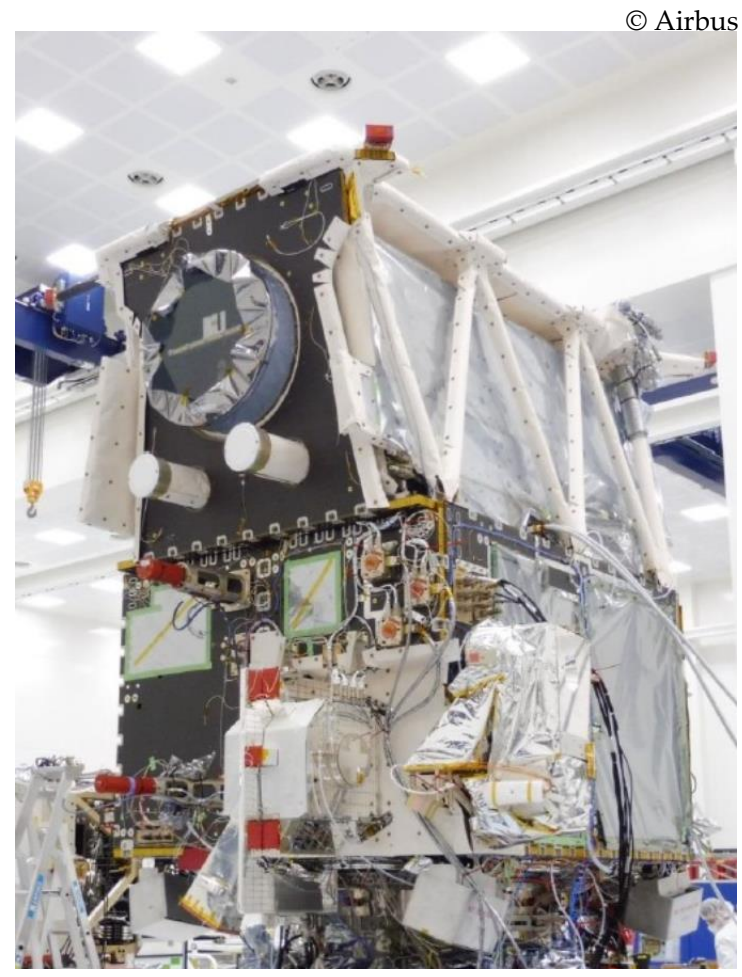

Figure 15. ATLID integrated on EarthCARE satellite platform.

\section{References}
1. EarthCARE
- Clouds,
Aerosols
and
Radiation
Explorer,

http://esamultimedia.esa.int/docs/SP_1279_1_EarthCARE.pdf 
2. Lefebvre, A., et al: "EarthCARE mission, overview, implementation approach and development status," Proc. SPIE 9881, 2016

3. Hélière, A., et al: "Earth Cloud, Aerosol and Radiation Explorer optical payload development status", Proc. SPIE 10423, 2017

4. Lefebvre, A., et al: "EarthCARE mission: approaching launch”, IEEE International Geoscience and Remote Sensing Symposium (IGARSS) 2018

5. Lefebvre, A., et al: "Status of ESA's EarthCARE Mission Preparation", IEEE International Geoscience and Remote Sensing Symposium (IGARSS) 2020

6. Hélière, A., et al: "A Development status of the EarthCARE Mission and its atmospheric Lidar," Proc. SPIE 9972-42, 2017

7. Pereira do Carmo, J., et al: “ATLID, ESA atmospheric lidar : manufacture and test results of instrument units", International Conference on Space Optics (ICSO) 2016.

8. de Villele, G., at al: “ATLID, ESA Atmospheric LIDAR: integration of instrument and tests”, International Conference on Space Optics (ICSO) 2018

9. Pereira do Carmo, J., et al: "ATLID, ESA Atmospheric backscatter LIDAR for the ESA EarthCARE Mission", Council of European Aerospace Societies (CEAS) Space Journal 11, pp. 423-435, 2019

10. Pereira do Carmo, J, et al: "Flight LIDAR development and qualification for the ESA Earth Cloud Aerosol and radiation explorer (EarthCARE) mission", IEEE International Geoscience and Remote Sensing Symposium (IGARSS) 2020

11. Wallace, K., et al: "Status of ESA's EarthCARE mission, passive instruments payload", Proc. SPIE 9972-41, 2017

12. Tomiyama, N., et al: "EarthCARE/CPR development status and performance", Proc. SPIE Proceedings Vol. 11531 Remote Sesning of Clouds and Atmosphere XXV, 2020

13. Straume, A. G., et al.: "ESA's space-based Doppler wind lidar mission Aeolus -First wind and aerosol product assessment results", EPJ Web Conf., 237, 01007, https://doi.org/10.1051/epjconf/202023701007, 2020.

14. Weiler, F., et al, "Characterisation of dark current measurements of the ACCDs used on-board Aeolus satellite", Journal - Atmospheric Measurement Techniques, (submitted), 2020 